\title{
Assessment of the Glycotronic C blood glucose meter
}

\author{
A R W Forrest, E J Pitt
}

\begin{abstract}
The performance of the Glycotronic C blood glucose meter, a pocket sized glucose strip meter, was assessed on the ward and in the laboratory. Theoretically there is a relation between blood glucose concentration, plasma glucose concentration and haematocrit when glucose is measured using conventional methods. The Glycotronic C meter would yield results about $20 \%$ greater than those of a conventional instrument when used to measure plasma glucose and about $15 \%$ less than a conventional instrument when used to measure the blood glucose concentration of a neonate. The Glycotronic $C$ is easy to use and gives an acceptable performance for near patient use; it compares well with its current British competitors.
\end{abstract}

\section{Methods}

Blood glucose analyses were carried out using the Glycotronic $\mathrm{C}$ instrument and a Yellow Springs Instruments YSI 23AM glucose analyser (Clandon Scientific Ltd, Aldershot, England). Blood samples were either anticoagulated samples or capillary samples collected by ward nursing staff. In the former, the sample was analysed simultaneously using the YSI 23AM and the Glycotronic C; in the latter, an aliquot was analysed immediately using the Glycotronic $\mathrm{C}$ and the remainder of the sample was collected into a tube containing fluoride oxalate preservative and analysed within an hour with the YSI 23AM. The effect of sample haematocrit on analytical performance was assessed by centrifuging a sample of anticoagulated blood, separating the red cells and plasma, and preparing a series of dilutions of the red cells with the plasma, using a positive displacement pipette. As each dilution of known haematocrit was prepared it was at once analysed using both the YSI 23AM and Glycotronic C.

Analytical precision was assessed by using paired replicate analysis to calculate the coefficient of variation (CV). The results of analyses carried out on samples with the Glycotronic C were compared with those performed using the YSI 23AM by the methods described by Bland and Altman. ${ }^{1}$
A total of 53 samples were analysed in duplicate using the Glycotronic $\mathrm{C}$. The mean glucose concentration was $6.9 \mathrm{mmol} / 1$ with an assay $\mathrm{CV}$ of $5 \cdot 2 \%$. Seventy three samples collected on the ward were analysed by nursing staff using the Glycotronic $C$ and laboratory staff using the YSI 23AM. The results produced by the Glycotronic $C$ showed a negative bias of $0.44 \mathrm{mmol} / 1(95 \%$ confidence interval 0.28 to $0.60 \mathrm{mmol} / \mathrm{l}$ ), when compared with those generated by the YSI 23AM. The performance of the Glucotronic $\mathrm{C}$ is comparable with that reported for other reflectance meters. ${ }^{2}$

When samples of varying haematocrit, prepared as described above, were analysed, the Glycotronic $\mathrm{C}$ produced results that were higher than those given by the YSI 23AM on the same sample when the haematocrit was low, and lower than the YSI 23AM results when the haematocrit was high (fig 1).

\section{Discussion}

The Glycotronic $\mathrm{C}$ is a compact and easy to use instrument. With a minimum of instruction, nursing and laboratory staff quickly learned to produce results that, although not as precise as can be expected from a laboratory, ${ }^{3}$ are certainly adequate for many clinical purposes. Only one instrument was available for evaluation, and the tendency to produce results about $4 \%$ lower than those produced by the YSI 23AM may not be common to all instruments.

The effect of haematocrit on the blood glucose results generated by instruments measuring blood glucose merits some consideration. Clearly, when red cells are in equilibrium with plasma glucose-for exam-

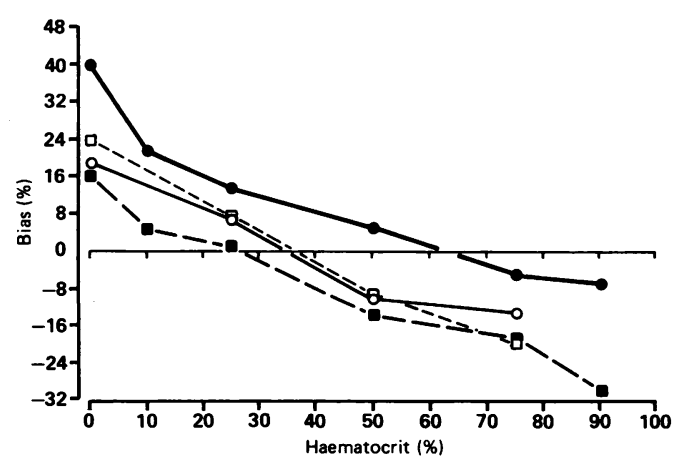

Figure 1 Effect of varying haematocrit on the difference between the Glycotronic $C$ and the YSI 23AM result, expressed as a percentage of the difference between the Glucotronic $C$ and the $Y S I 23 A M$ result with respect to the YSI $23 A M$ result.

(([Glucotronic $]-[$ YSI $]) .100) /(Y S I]) \%$ 
ple, when preserved with fluoride, the concentration of glucose per unit volume of red cells will be lower than the concentration of glucose per unit volume of plasma, for if the glucose is mainly distributed in the plasma and cellular water, then there is less water per unit volume of red cell than per unit volume of plasma.

If $\mathrm{H}=$ haematocrit (expressed as a fraction), $\mathrm{C}=$ red cell glucose concentration, $\mathrm{p}=$ plasma glucose concentration, $\mathrm{w}=$ whole blood glucose concentration, then when the plasma glucose and red cell glucose concentrations are constant and the haematocrit varies, we can write:

$$
\begin{aligned}
& \mathbf{R}=\mathbf{C} / \mathbf{P} \\
& \mathbf{W}=\mathbf{C} . \mathbf{H}+\mathbf{P} .(1-\mathbf{H})
\end{aligned}
$$

Equation 1

Combining equations 1 and 2 yields

$$
\mathrm{W} / \mathbf{P}=\mathbf{1}-\mathbf{H} .(1-\mathbf{R}) \quad \text { Equation } 3
$$

That is to say, the ratio of the whole blood glucose to the plasma glucose concentration is a function of both the haematocrit and the ratio (" $R$ ") of the red cell glucose concentration to the plasma glucose concentration.

Instruments such as the YSI 23AM in which glucose is analysed in an environment that disrupts the red blood cells in the sample will generate results that closely follow those predicted by equation 3 as the haematocrit varies (fig 2). Enzyme strip based analysers will essentially measure the glucose in the plasma phase of blood. Glucose present inside red cells will only have restricted access to the

Figure 2 The theoretical and observed relation between whole blood glucose concentration, expressed as a proportion of the plasma glucose concentration, when the haematocrit varies and the plasma and red cell glucose concentration stay constant. The blood glucose concentration was measured with the YSI $23 A M$. strip surface while the blood sample is in contact with the strip. Thus when the haematocrit is high, less plasma will have access to the strip surface, as a higher proportion of the strip will have red blood cells rather than plasma in contact with it, and a lesser degree of colour development will take place than when the haematocrit is low. So, not only will blood glucose results from enzyme strip instruments only agree with the results obtained from blood glucose analysers when the haematocrit in the sample is around the normal, when the haematocrit differs significantly from normal the deviance from the expected result will be greater than that predicted by equation 3 . Thus a high result will be obtained if an enzyme strip is used to measure plasma and a low result will be obtained if the haematocrit is high. This effect may be clinically important; the Glycotronic $\mathrm{C}$ could be expected to give results some $20 \%$ higher than those produced by a YSI 23AM if used to measure a plasma sample and some $15 \%$ lower than those produced by a YSI 23AM when used to measure glucose in a sample from a new born infant (fig 1). The haematocrit effect is not, of course, unique to the Glycotronic C. Any glucose analyser that uses a whole blood sample without disruption of the red cells can be expected to show the same phenomenon.

Overall, the Glycotronic $\mathrm{C}$ is a pleasant and easy to use instrument, with a performance that is acceptable for most near patient work. It compares well with its current competitors in the British market.

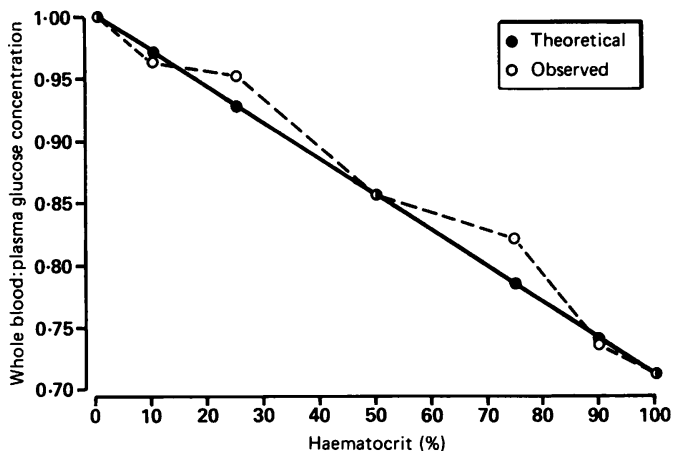

We are grateful to the nursing staff of Ward P1 of the Royal Hallamshire Hospital for their help. The Glycotronic C meter and test strips used were supplied by Cambridge Self Care Diagnostics Ltd, Newcastle upon Tyne.

1 Bland JM, Altman DG. Statistical methods for assessing agreement between two methods of clinical measurement. agreement between tw

2 Begley JP, Forrest ARW. Laboratory assessment of three reflectance meters designed for self monitoring of blood glucose concentrations. J Clin Pathol 1988;41:397-402.

3 Fraser CG. Analytical goals for glucose analyses. Ann Clin Biochem 1986;23:379-89. 